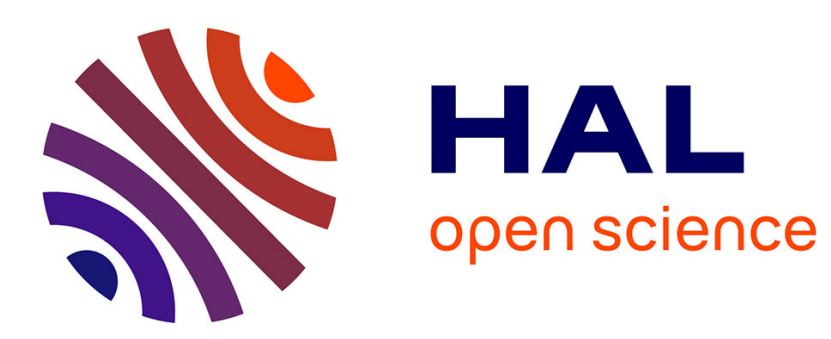

\title{
Secure and Efficient Pseudonymization for Privacy-Preserving Vehicular Communications in Smart Cities
}

Siham Bouchelaghem, Mawloud Omar

\section{- To cite this version:}

Siham Bouchelaghem, Mawloud Omar. Secure and Efficient Pseudonymization for Privacy-Preserving Vehicular Communications in Smart Cities. Computers and Electrical Engineering, 2020. hal03033609

\section{HAL Id: hal-03033609 \\ https://hal.science/hal-03033609}

Submitted on 1 Dec 2020

HAL is a multi-disciplinary open access archive for the deposit and dissemination of scientific research documents, whether they are published or not. The documents may come from teaching and research institutions in France or abroad, or from public or private research centers.
L'archive ouverte pluridisciplinaire HAL, est destinée au dépôt et à la diffusion de documents scientifiques de niveau recherche, publiés ou non, émanant des établissements d'enseignement et de recherche français ou étrangers, des laboratoires publics ou privés. 


\title{
Secure and Efficient Pseudonymization for Privacy-Preserving Vehicular Communications in Smart Cities
}

\author{
Siham Bouchelaghem and Mawloud Omar \\ Unité de Recherche LaMOS, Faculté des Sciences Exactes \\ Université de Bejaia, 06000 Bejaia, Algérie.
}

\begin{abstract}
The emergence of modern Intelligent Transportation Systems (ITS) based on vehicular networks (VNs) has diversified the range of applications provided to drivers and passengers during their road trips. To fully benefit from these services, vehicles need however to share their geolocation data making location privacy a critical issue. Hence, to mitigate these threats and promote VNs use in developing Smart Cities, it is vital to guarantee conditional anonymity to vehicular users. In this paper, we propose an efficient pseudonym changing strategy for privacy-preserving in VNs. The proposed approach uses threshold cryptography properties to provide moving vehicles with pseudonyms, allowing them to communicate anonymously during their journey. These vehicles though can be traced back by trusted authorities in case of misbehavior actions. We evaluate the efficiency of the proposed approach by comparison with concurrent solutions, where it demonstrates the best results in both congested road traffic and dense infrastructure deployment scenarios.
\end{abstract}

Keywords: Vehicular networks, Conditional anonymity, Pseudonym changing, Threshold cryptography, Privacy.

\section{Introduction}

Driving assistance, location-based services and route guidance are the main requirements for the design of efficient traffic management systems in Smart Cities [1, 2]. As part of the Intelligent Transportation Systems (ITS), vehicular networks (VNs) are expected to revolutionize the development of promising applications to provide citizens with a safer driving environment and a more convenient traveling experience on the roads. These applications can be ranging from safety enhancing through collision warning and emergency reporting, to non-safety applications such as location-based services for finding nearby places, route reporting for traffic management, and infotainment. To enable these various applications of VNs, vehicles are currently equipped with navigation systems and have wireless communication capabilities. Despite their wide deployment, the open nature of VNs still raises some serious security issues, which should be addressed [3, 4. In fact, people are increasingly worried about the impact this technology will have on their privacy. For instance, an adversary eavesdropping on the communications can use sensitive information (e.g., geolocation data, velocity) to track a specific vehicle, and profile its driver movements and life pattern. These 
malicious actions may not only threaten citizens privacy, but also lead to physical harm and attempted robberies. To address such privacy issues, both the research community and the automotive industry have agreed on using pseudonyms to provide VN users with the required protection [5. However, designing an effective pseudonym changing strategy that copes with the inherent characteristics of VNs is still a challenging issue. In this context, several solutions for location privacy in VNs have been proposed in the literature. Most of the existing solutions relies on the unique network characteristics such as traffic density, mobility criteria, and strategic geographical areas to allow vehicles pseudonym change. However, these solutions present some limitations as they lack flexibility, are less efficient in low density situations, and may affect safety level and liability requirements.

In this paper, we propose a pseudonym changing strategy for privacy-preserving communications in VNs. The proposed approach is designed to provide location privacy to all VN participants, which can communicate anonymously during their trips. Unlike the existing solutions of the literature, where each vehicle must change pseudonym at a specific place, the proposed approach meets the network constraints and does not rely on spatial or temporal dependencies. Moreover, the proposed approach overcomes the traffic density requirements of the existing solutions as any vehicle on the road can autonomously change its pseudonym without waiting to have a certain number of vehicles in the vicinity. The proposed approach relies on Vehicle-to-Infrastructure (V2I) communication to perform the pseudonym changing strategy as each vehicle moving along the road section can request a new pseudonym from the deployed Road-Side Units (RSUs). As the next generation of ITS, Smart Cities tend to leverage V2I technology for urban mobility promising revolutionary improvements in transportation. In fact, infrastructure plays a key role in the Smart Cities paradigm especially when relating to VNs as it ensures traffic information gathering and value-added services deployment for real-time drivers warning about unsafe road conditions and imminent collisions, traffic congestion, construction zones and parking lots availability, etc. Moreover, since Vehicle-to-Vehicle (V2V) communication could suffer reliability issues under certain conditions and areas, current Smart Cities are vastly equipped with infrastructures to improve such systems performances by combining V2I communications [6, 7, 8, 9]. The proposed approach is performed under a $\langle t, n\rangle$ threshold encryption scheme and designed in three distinct phases namely, (1) system registration, (2) pseudonym generation, and (3) pseudonym verification and recovery. The first phase is about vehicles registration and the initialization of some cryptographic parameters. The second phase consists in assigning a new pseudonym to each requesting vehicle on the road. Finally, the third phase is about vehicles pseudonym verification, and their real identities disclosure in case of misbehavior. Through the security analysis, we demonstrate the robustness of the proposed approach against some well-known attacks. Moreover, we evaluate the performances of the proposed solution, which we compare to the reviewed approaches. In fact, we have developed simulations, in which we highlight the advantages of the proposed approach in terms of storage load, communication overhead and response time.

The rest of the paper is organized as follows. In Section 2, we review some relevant solutions designed for location privacy-preserving in VNs. In Section 3 , we give the detailed description of the proposed approach. In Section 4 , we analyze the security of 


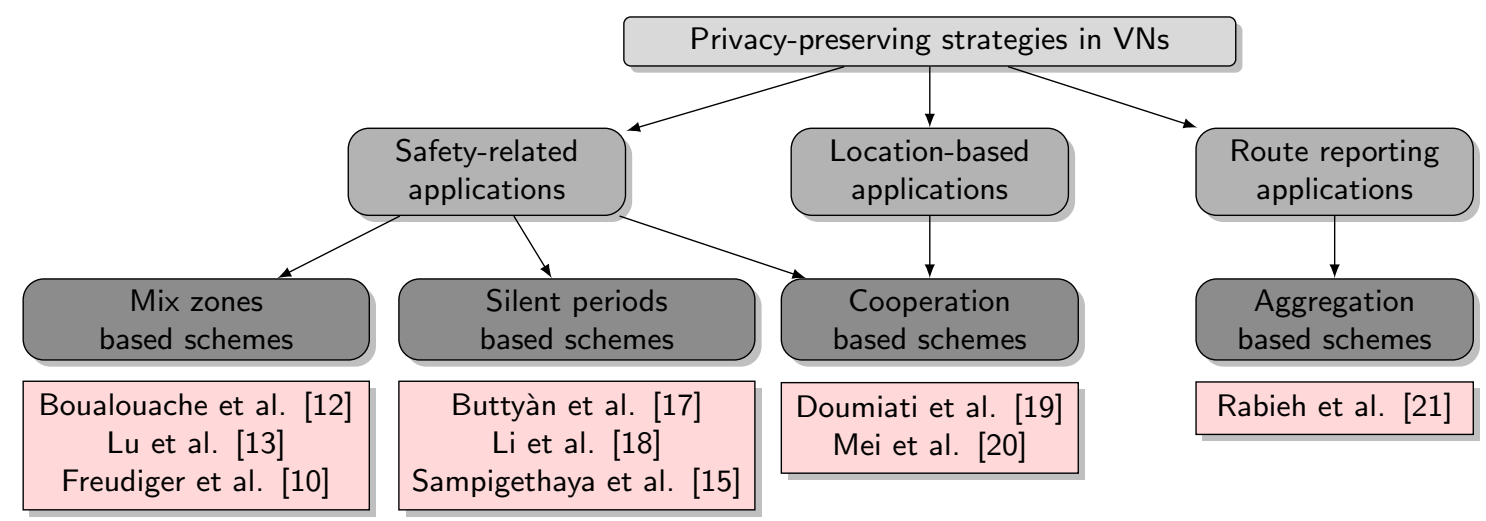

Figure 1: Classification of existing solutions

the proposed approach, and in Section 5, we evaluate its efficiency through intensive simulations. Finally, we conclude the paper in Section 6 .

\section{Related work}

In the literature, various approaches have been investigated to preserve drivers privacy in different applications of VNs. In this section, we review and compare some relevant solutions, which we classify as illustrated in Figure 1.

Freudiger et al. [10] have proposed to create CMIX zones (Cryptographic MIX zones) at road intersections to achieve vehicles location privacy while assuring vehicular safety services. Mix zones are anonymizing regions where vehicles change their pseudonyms. They are usually considered as regions that cannot be controlled by an adversary [11]. However, since mix zones are placed at predetermined locations, this latter can easily identify them and attempt to eavesdrop the transmissions within those regions. Hence, to prevent an adversary from monitoring safety messages, all legitimate vehicles obtain a symmetric key from the RSU of the mix zone to encrypt their messages until leaving the zone. Still, an internal adversary can have the decryption key and disclose the information contained in safety messages. Besides, the processing time overhead to perform the cryptographic operations does not meet with real time constraints of safety-related applications. Boualouache et al. [12] have proposed a protocol based on VLPZ (Vehicular Location Privacy Zone), a roadside infrastructure designed to change vehicles pseudonyms and protect their location privacy. VLPZs are managed by trusted regional authorities and can be implemented in existing roadside infrastructures such as gas stations, toll booths, etc. VLPZs consist of an entry point called router, a limited number of lanes, and an exit point called aggregator. When a vehicle enters a VLPZ from the router, it stops broadcasting safety messages, heads up to its assigned lane and changes its pseudonym before it exits the VLPZ by the aggregator. The assigned lane is randomly and privately selected by the router. Lu et al. [13] have proposed to place mix zones at specified locations called social spots, which are public places areas such as signalized intersections, free parking lots near a shopping mall, etc. Vehicles gathering in front the red traffic light at a signalized intersection, change their pseudonyms when the traffic light turns green. Similarly, a vehicle that 
stops at a free parking lot near a mall, changes its pseudonym before leaving the parking lot. The use of mix zones has demonstrated potential to preserve location privacy, since several vehicles changing their pseudonym at the same place make an external adversary confused. However, solutions based on mix zones lack flexibility as they rely on predetermined locations. Privacy protection level also depends on traffic density and the unpredictable nature of VNs. Moreover, selfish vehicles may not change their pseudonyms because of limited resources. This could jeopardize the location privacy of those vehicles that are in need of changing their pseudonyms [14. Sampigethaya et al. 15] have proposed CARAVAN a location privacy scheme based on random silent periods techniques to mitigate vehicles tracking. In fact, a vehicle remains silent (i.e., stops sending safety messages) for a randomly chosen period of time after changing its pseudonym. By combining group concept, vehicles can be provided with extended silent periods as only the group leader needs to communicate on behalf of the group. However, in case of group leader collusion with an adversary, the anonymity of group members can be breached. Hence, the authors have proposed to use a group key and an application address range to prevent trace back from group leader to a given group member in any identifiable area. In fact, since the random address used is not associated with the vehicle, exchanged requests cannot be associated with any of its pseudonyms. So far, group communication and data exchange techniques have gained much interest for providing value-added services in VNs under privacy preserving [16]. Buttyán et al. [17] have proposed SLOW (Silence at LOW speeds) a protocol that uses radio silence concept to prevent vehicles traceability and ensure drivers location privacy. Vehicles stop sending safety messages when they are traveling below a predetermined threshold speed (e.g., less than $30 \mathrm{~km} / \mathrm{h}$ ) and change their pseudonyms during such silent periods. Li et al. 18] have proposed two protocols Swing and Swap enabling vehicles coordinate their silent periods and decide whether to change pseudonyms. In Swing, vehicles update pseudonyms only when changing their direction and speed. Swap is an extension of Swing, where vehicles exchange their pseudonyms with each other and then enter a random silent period. Swap maximizes location privacy by including even non-updating neighboring vehicles, however, each vehicle that changes its pseudonym is only indistinguishable from the vehicle requesting exchange, and not from the other vehicles. Moreover, exchanging pseudonyms in VNs does not meet with vehicles liability requirements. Doumiati et al. [19] have proposed LPS for LBS, a Location Privacy protection Scheme for LBS (Location-Based Services). LBS help vehicular users obtaining information on static resources such as the nearest banking cash machine, fuel station, restaurant and touristic places. To answer a driver request and provide its services, the LBS uses critical information such as vehicle location, its speed, license plate, etc. Thus, the LBS is considered as an untrusted third party, which can profile drivers interests and track them until their destination. Based on this, the authors have proposed to rely on group communication concept to preserve drivers privacy while using LBS. A request addressed to the LBS is encrypted with several public keys where only the entities having the corresponding private keys can decrypt it. Moreover, group communication ensures that only a group leader communicates with the LBS on behalf of the group, which obfuscates the source of the request. Mei et al. 20] have proposed a collaboratively hidden location privacy scheme, which uses a variant of ring signature to authenticate safety messages and ensure vehicles location privacy. A vehicle can form a ring arbitrar- 
ily with surrounding vehicles and sign its messages anonymously. To form a ring with nearby vehicles and find their public keys, each vehicle sends a STM (Signed Timestamp Message) signed with a ring that just holds the signer public key. The authors have proposed two approaches for ring formation in two different situations. In the centralized approach, vehicles broadcast STMs in predetermined social spots with the help of RSUs. Whereas in the distributed approach, vehicles can dynamically choose time and location to broadcast STMs with the cooperation of neighboring vehicles in case of nonsocial spots situations. In general, solutions based on vehicles cooperation cannot be applied in lower traffic density areas. Moreover, waiting to have the required number of vehicles in the vicinity may degrade the quality of service of real time applications such as LBSs and safety driving services. Rabieh et al. [21] have proposed $\mathrm{P}^{2} \mathrm{R}^{2} \mathrm{TM}$, a Privacy-Preserving Route Reporting scheme for Traffic Management systems. Route guidance enables drivers to avoid potential congestion and pursue alternative routes by informing them about the expected traffic information. However, it requires that each vehicle reports its future locations, which can be used by adversaries not only to violate drivers privacy but also for physical attacks and robbery. Using homomorphic encryption, the authors have proposed to encrypt all the segments in a vehicle future route in one message, instead of encrypting each individual segment. Moreover, RSUs aggregate vehicles messages and send them to the TMC (Traffic Management Center), which prevents adversaries from knowing the individual vehicles routes. However, this type of solutions work better in high vehicle density scenarios.

\section{The proposed approach}

In this section, we present the system model and detail the proposed approach operations.

\subsection{System model}

We consider a VN, where each vehicle is equipped with an On-Board Unit (OBU) to communicate with other vehicles and with RSUs deployed along the road. The communication that takes place between vehicles by using the OBU is known as V2V communication. We assume that each vehicle has a GPS (Global Positioning System) device to geolocalize itself and obtain its position and current time. Vehicles can communicate with the Trusted Authority (TA) through the neighboring RSUs forming a V2I communication (cf., Figure 2). Each vehicle in the network periodically broadcasts safety messages or requests location-based services to share local traffic information and make the driving conditions safer and more convenient.

We consider a threat model where a passive external adversary can eavesdrop the network and infer sensitive information from the exchanged safety messages. Such an eavesdropper may try to link several vehicle pseudonyms, and manage to figure out the real identity of a target vehicle. An adversary may also analyze the geolocation data through the safety messages broadcast by the OBU. Such sensitive information may be used to track a specific vehicle, and profile the driver movements and life pattern such as his home address, place of work, etc. On the other hand, an internal adversary may benefit from the anonymity to run out from sanctions in case of misbehavior (e.g., sending false information about the road conditions to create traffic jams and cause 


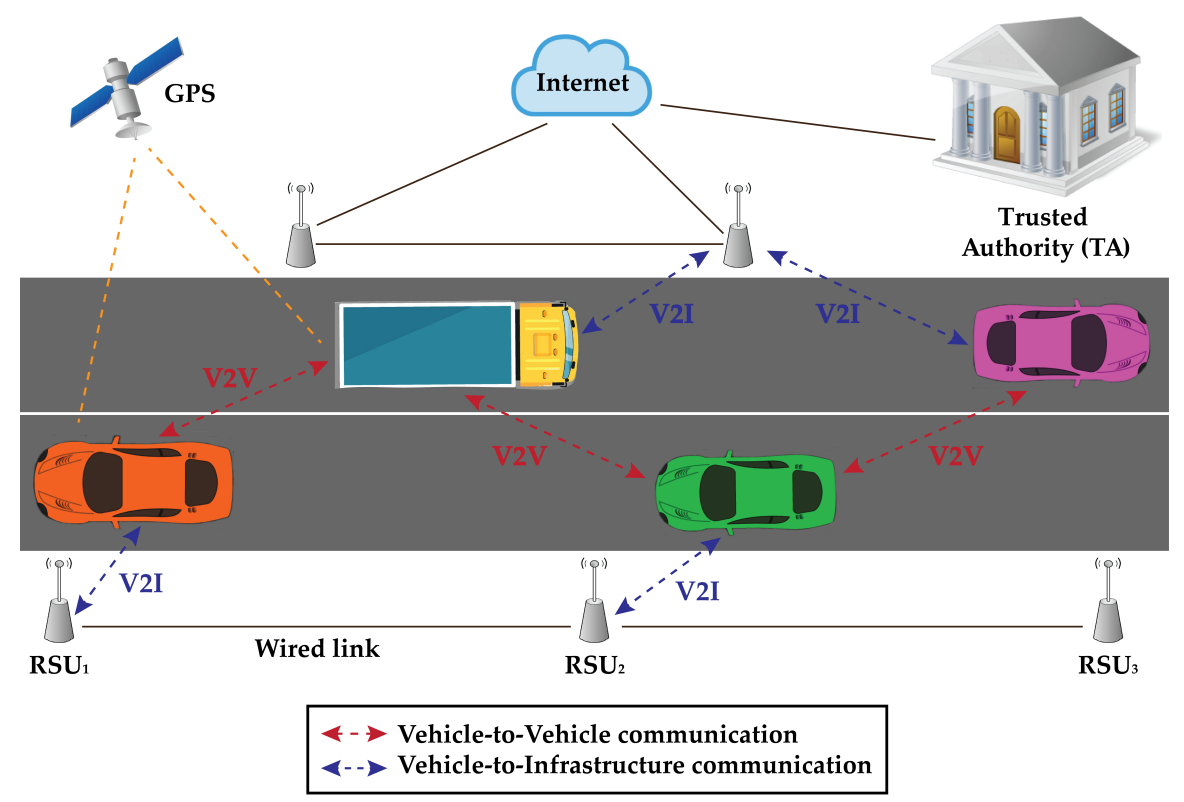

Figure 2: Overview of VN architecture

accidents). We assume that the TA is a fully trusted third party, which does not reveal the real vehicles identities, as it is likely to belong to a governmental organization such as the Department of Transportation. Finally, we note that physical attacks, where a compromised party gain access to the OBU of vehicles, as well as traffic monitoring cameras-based tracking are beyond the scope of this paper. We summarize the main used notations in Table 1 .

\begin{tabular}{|c|l|}
\hline Notation & Description \\
\hline \hline$v_{i}$ & Vehicle of identity $i$ \\
\hline$R S U_{j}$ & Road-Side Unit of identity $j$ \\
\hline$P_{i}^{(0)}$ & Vehicle $v_{i}$ initial pseudonym pool \\
\hline$p_{i k}^{(0)}$ & $k^{t h}$ vehicle $v_{i}$ initial pseudonym \\
\hline$p_{i}^{(j)}$ & Vehicle $v_{i}$ new pseudonym generated by $R S U_{j}$ \\
\hline$K_{j}, \widehat{K}_{j}$ & Road-Side Unit $R S U_{j}$ public and private keys \\
\hline$e_{k}^{(j)}, d_{k}^{(j)}$ & Road-Side Unit $R S U_{j}$ encryption and decryption shares \\
\hline$e_{k}, d_{k}$ & Group encryption and decryption keys \\
\hline$\left\langle L_{i}, T_{i}\right\rangle$ & Geolocation tuple of vehicle $v_{i}$ \\
\hline$\langle t, n\rangle$ & Threshold-based encryption scheme \\
\hline $\mathcal{H}$ & Collision-resistant hash function \\
\hline$F_{p}$ & Finite field of cardinal $p$ \\
\hline$E_{p}(a, b)$ & Elliptic curve over $F_{p}$ \\
\hline$Q$ & Base point on the elliptic curve \\
\hline$q$ & Large prime number \\
\hline
\end{tabular}

Table 1: Notations 


\subsection{System registration phase}

Before starting the pseudonym changing process, the TA should bootstrap the whole system and initialize some cryptographic parameters. For instance, each vehicle will have a unique identifier $v_{i}$, which will be bound to its pseudonyms. This correspondence will be known only by the TA. To do so, it will generate for each vehicle $v_{i}$ an initial pool of $N$ pseudonyms $P_{i}^{(0)}$ such as $P_{i}^{(0)}=\left\{p_{i 1}^{(0)}, p_{i 2}^{(0)}, \cdots, p_{i N}^{(0)}\right\}$. These pseudonyms will be initially used by a vehicle $v_{i}$ at each newly trip, and are computed such as

$$
p_{i k}^{(0)}=\mathcal{H}\left(i\left\|I D_{T A}\right\| T_{\text {exp }}\left\|T_{s}\right\| r_{k}\right), \quad k=1 . . N
$$

where $\mathcal{H}$ is a collision-resistant hash function, $T_{\text {exp }}$ is the pseudonym expiration time to reduce the leaking risk and enhance the privacy protection, $T_{s}$ a timestamp to prevent replay attacks, and $r_{k}$ a random number to increase the variability in the resulting pseudonyms and mitigate brute force attacks.

The TA also needs to generate for each roadside unit $R S U_{j}$ a public and private key pair $\left\langle K_{j}, \widehat{K}_{j}\right\rangle$, which is bound to the RSU identifier and will be used to securely communicate with the TA.

\subsection{Pseudonym generation phase}

When a vehicle $v_{i}$ starts a trip, it randomly chooses its first pseudonym $p_{i k}^{(0)}$ from the pseudonym pool embedded in its OBU. Upon belonging in the communication range of the first RSU in a given location $L_{i}$ at a given time $T_{i}$, the vehicle $v_{i}$ sends a request $R e q=\left\langle p_{i k}^{(0)}, L_{i}, T_{i}\right\rangle$ to ask for a new pseudonym, which will be used for any vehicular communication along this route section. The initiator RSU transmits then the vehicle request to the TA, which will share a secret encryption key through a $\langle t, n\rangle$ threshold scheme based on ECC (Elliptic Curve Cryptography) as depicted in Figure 3. We design the encryption key distribution and pseudonym generation process on the threshold signature scheme proposed in [22]. The detailed operations are described in the following subsections.

\subsubsection{Threshold-based encryption key distribution}

First, the TA chooses an elliptic curve $E_{p}(a, b)$ over a finite field $F_{p}$, and a base point $Q$. Then, it generates a secret encryption key $e_{k} \in[1, q-1]$, computes its corresponding decryption key $d_{k}$ such as

$$
d_{k}=e_{k} \cdot Q
$$

and publishes the public parameters $p, q, E_{p}(a, b)$ and $Q$, while $d_{k}$ is kept secret. Afterwards, it randomly chooses a secret polynomial $f$ of degree $t-1$ such as

$$
f(x)=a_{0}+a_{1} x+\cdots+a_{t-1} x^{t-1} \bmod q,
$$

where

$$
f(0)=a_{0}=e_{k} .
$$




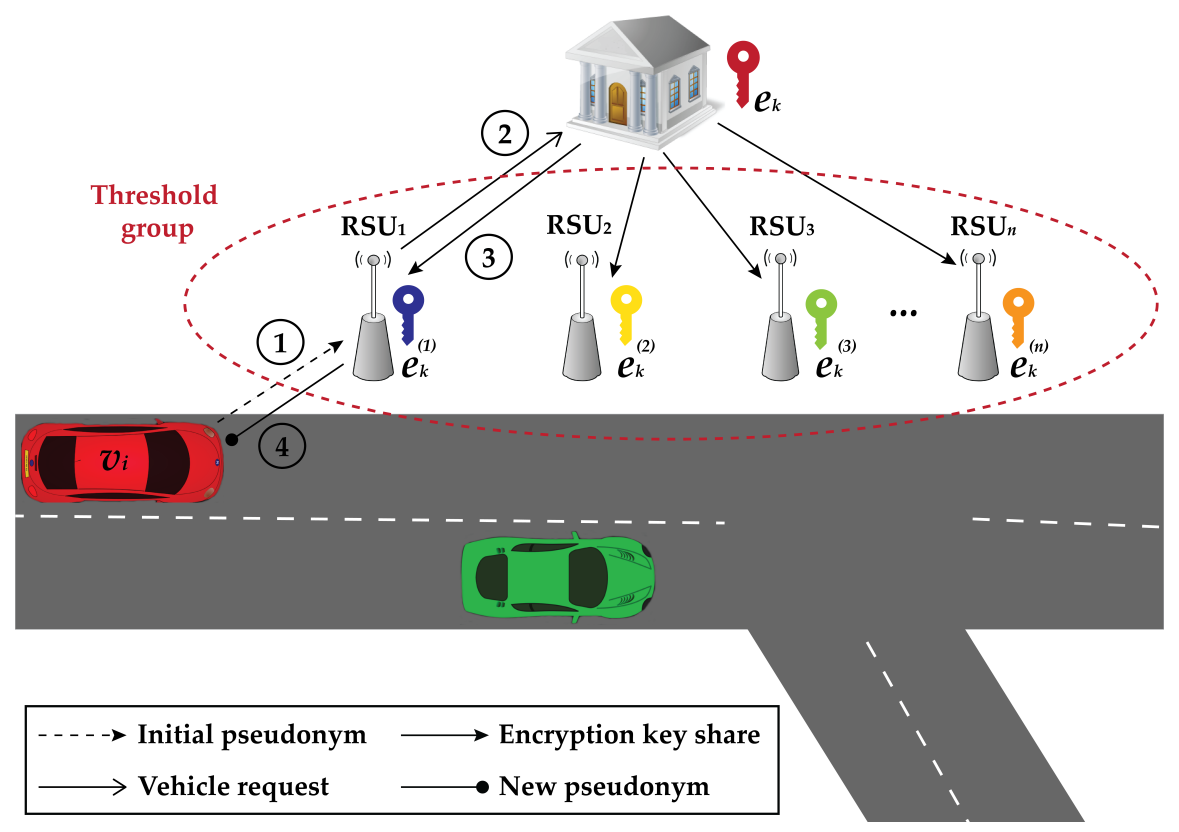

Figure 3: Threshold-based pseudonym changing strategy

Next, it computes for each roadside unit $R S U_{j}$ along the route section, a secret encryption share $e_{k}^{(j)}$ such as

$$
e_{k}^{(j)}=f(j)
$$

and stores the corresponding decryption shares $d_{k}^{(j)}$ such as

$$
d_{k}^{(j)}=e_{k}^{(j)} \cdot Q
$$

The TA transmits then the secret encryption shares $e_{k}^{(j)}$ to each roadside unit $R S U_{j}$ encrypted with their respective public keys. To securely send the secret shares $e_{k}^{(j)}$, the TA computes $C_{1}^{(j)}$ and $C_{2}^{(j)}$ such as

$$
C_{1}^{(j)}=e_{k}^{(j)}+\alpha^{(j)} \cdot K_{j}
$$

and

$$
C_{2}^{(j)}=\alpha^{(j)} \cdot Q
$$

where $\alpha^{(j)}$ are randomly selected integers and $K_{j}$ the public key of $R S U_{j}$.

Finally, the TA sends $\left\langle C_{1}^{(j)}, C_{2}^{(j)}\right\rangle$ to each $R S U_{j}$, which uses its private key $\widehat{K}_{j}$ to recover its secret encryption share $e_{k}^{(j)}$. To do this, it computes

$$
e_{k}^{(j)}=C_{1}^{(j)}-\widehat{K}_{j} \cdot C_{2}^{(j)} .
$$




\subsubsection{Individual pseudonym encryption}

Upon receiving its secret encryption share $e_{k}^{(j)}$, each roadside unit $R S U_{j}$ randomly selects a number $c_{j} \in[1, q-1]$ and computes $Z_{j}$ such as

$$
Z_{j}=c_{j} \cdot Q
$$

The set of $Z_{j}$ are sent over a secure communication channel to all neighboring RSUs along the route section, and each one of them computes $Z$ such as

$$
Z=\sum_{j=1}^{n} Z_{j},
$$

where $n$ is the number of neighboring RSUs along the route section.

Each time the vehicle $v_{i}$ passes by a roadside unit $R S U_{j}$ along the route section, it will send its first pseudonym $p_{i k}^{(0)}$ to $R S U_{j}$, which computes the hash value $h$ such as

$$
h=\mathcal{H}\left(p_{i k}^{(0)} \| x_{Z}\right)
$$

where $x_{Z}$ is the $x$-coordinate of the point $Z$.

Afterwards, the roadside unit $R S U_{j}$ computes $x_{j}$ and $u_{j}$ such as

$$
x_{j}=\prod_{i=1, i \neq j}^{n} \frac{-i}{(j-i)} \bmod q,
$$

and

$$
u_{j}=e_{k}^{(j)} \cdot x_{j} \bmod q
$$

Finally, the $R S U_{j}$ will generate a new pseudonym $p_{i}^{(j)}$ for vehicle $v_{i}$ such as

$$
p_{i}^{(j)}=c_{j}-u_{j} \cdot h \bmod q,
$$

and send $\left\langle I D_{R S U_{j}}, p_{i k}^{(0)},\left\{h, p_{i}^{(j)}, Z_{j}\right\}, L_{j}, T_{j}\right\rangle$ over a secure communication channel to the TA, which will keep a record of the pseudonym correspondence to the actual identity of the vehicle $v_{i}$. The new pseudonyms $p_{i}^{(j)}$ can be then used by the vehicle $v_{i}$ to send safety messages, request location-based services, enable traffic prediction and infotainment systems, etc.

\subsection{Pseudonym verification and recovery phase}

Despite the need to preserve vehicles location privacy, there are some application fields where their real identities need to be disclosed. Moreover, to meet with the requirement of maintaining liability of vehicles involved in accidents for instance, TAs must have the ability to link each pseudonym $p_{i}^{(j)}$ to the real identity of any vehicle $v_{i}$. Hence, to ensure conditional anonymity, TAs must verify the validity of each generated pseudonym and detect potential vehicles and RSUs collusion. To do so, upon receiving a new pseudonym $p_{i}^{(j)}$ from each $R S U_{j}$, the TA computes $Z_{j}^{\prime}$ such as

$$
Z_{j}^{\prime}=p_{i}^{(j)} \cdot Q+h \cdot x_{j} \cdot d_{k}^{(j)}
$$


and verifies the validity of the individual pseudonym $p_{i}^{(j)}$ encryption by

$$
Z_{j}=Z_{j}^{\prime}
$$

The TA chooses then $t$ individual pseudonyms encryption $p_{i}^{(j)}$ and computes $p_{i}$ such as

$$
p_{i}=\sum_{j=1}^{t} p_{i}^{(j)} \bmod q .
$$

Afterwards, it verifies the validity of the group pseudonym encryption $p_{i}$ by computing

$$
\begin{gathered}
\mathcal{G}=\sum_{j=1}^{t} Z_{j}, \\
\mathcal{G}^{\prime}=p_{i} \cdot Q+h \cdot d_{k}=\left(x_{\mathcal{G}^{\prime}}, y_{\mathcal{G}^{\prime}}\right),
\end{gathered}
$$

and

$$
h^{\prime}=\mathcal{H}\left(p_{i k}^{(0)} \| x_{\mathcal{G}^{\prime}}\right)
$$

If $\mathcal{G}=\mathcal{G}^{\prime}$ and $h=h^{\prime}$, then the pseudonyms $p_{i}^{(j)}$ are valid and the TA can link them to the vehicle $v_{i}$ identity. In fact, with the knowledge of any $t$ pairs of $\left(j, e_{k}^{(j)}\right)$, the TA can ensure the validity of the pseudonyms $p_{i}^{(j)}$, as if all the RSUs have strictly carried out the pseudonym generation process, then we have

$$
\begin{aligned}
\mathcal{G}^{\prime} & =p_{i} \cdot Q+h \cdot d_{k} \\
& =\sum_{j=1}^{t} p_{i}^{(j)} \cdot Q+h \cdot e_{k} \cdot Q \\
& =\sum_{j=1}^{t}\left(c_{j}-u_{j} \cdot h\right) \cdot Q+h \cdot e_{k} \cdot Q \\
& =\sum_{j=1}^{t} c_{j} \cdot Q-h \cdot \sum_{j=1}^{t} u_{j} \cdot Q+h \cdot e_{k} \cdot Q,
\end{aligned}
$$

where

$$
\sum_{j=1}^{t} u_{j}=\sum_{j=1}^{t} e_{k}^{(j)} \cdot \prod_{i=1, i \neq j}^{t} \frac{-i}{(j-i)}=f(0)=e_{k}
$$


Hence,

$$
\mathcal{G}^{\prime}=\sum_{j=1}^{t} c_{j} \cdot Q-h \cdot e_{k} \cdot Q+h \cdot e_{k} \cdot Q
$$

that is

$$
\mathcal{G}^{\prime}=\sum_{j=1}^{t} c_{j} \cdot Q=\sum_{j=1}^{t} Z_{j}=\mathcal{G} .
$$

Once the pseudonyms validity verified, the TA can link them to the vehicle $v_{i}$ identity. This correspondence will be used to bind future messages or requests with actual vehicle $v_{i}$ identity. In fact, the TA uses the initial pseudonym $p_{i k}^{(0)}$ received from the RSUs to recover the real identity of vehicle $v_{i}$ since it knows the initial pseudonym pool of each registered vehicle. Hence, if a vehicle has malicious behavior (e.g., trying to cover up its involvement in an accident, run out from toll road charges, etc.), the TA can reveal the real identity of the vehicle and trace its liability.

\section{Security analysis}

In this section, we analyze the security properties of the proposed approach, and give an overall analysis of its robustness against the well-known attacks.

\subsection{Pseudonyms linking}

The proposed approach ensures the two privacy aspects, namely, the anonymity and the unlinkability. In fact, for each vehicle traveling along a road section its actual identity is kept anonymous as messages sent with the VN capabilities only include a pseudonym. Moreover, two pseudonyms that belong to the same vehicle cannot be linked to each other. Indeed, an external adversary cannot follow vehicles pseudonym changing as vehicle $v_{i}$ will have a new pseudonym $p_{i}^{(j)}$ each time it passes by a roadside unit $R S U_{j}$. These pseudonyms are encrypted with the secret key share of each $R S U_{j}$ along the route section.

\subsection{Location tracking}

An external adversary can try to link the exchanged messages to a vehicle and use the geolocation information to infer sensitive details about the driving pattern of its driver and profile his personal habits. To prevent such attacks, the OBU of each vehicle $v_{i}$ is embedded with a pool of $N$ initial pseudonyms $\left\{p_{i k}^{(0)}\right\}_{k=1}^{N}$ during the registration phase, where each pseudonym $p_{i k}^{(0)}$ will be used at the start of each newly trip. Hence, any adversary that eavesdrops the communications and learns the first pseudonym $p_{i k}^{(0)}$ of the vehicle $v_{i}$ cannot link it to other pseudonyms and track the vehicle movements as conditional unlinkability is ensured for any different executions of the proposed approach. 


\subsection{Collusion and unforgeability}

During driving, a vehicle $v_{i}$ may try to collude with a roadside unit $R S U_{j}$ within its communication range to avoid countermeasures after a misbehavior for instance. In fact, a compromised $R S U_{j}$ may provide vehicle $v_{i}$ with a forged pseudonym to cheat on the TA. To prevent such attack, the TA can verify the validity of the individual pseudonym encryption $p_{i}^{(j)}$ sent by $R S U_{j}$ through the equation $Z_{j}=p_{i}^{(j)} \cdot Q+h \cdot x_{j} \cdot d_{k}^{(j)}$. It is thus

infeasible to find a false $p_{i}^{(j)}$ which makes the latter equation holds. Moreover, even a set of $t-1$ or less neighboring RSUs along the route section cannot collude to generate false pseudonyms to vehicle $v_{i}$, as the TA can verify the group pseudonym encryption from any $t$ combination of $p_{i}^{(j)}$.

\subsection{Traceability and liability}

If a vehicle has a malicious behavior, then the TA should have the ability to trace and reveal its real identity. With the binding property of the threshold pseudonym generation scheme, each pseudonym $p_{i}^{(j)}$ can be traced back to a specific vehicle $v_{i}$. In fact, the TA can verify the validity of the generated pseudonyms using the decryption key $d_{k}$, and link them to the first pseudonym $p_{i k}^{(0)}$ used by the vehicle $v_{i}$. The TA can then recover its actual identity as it has recorded the correspondence between all the first pseudonyms $p_{i k}^{(0)}$ and the real identity of vehicle $v_{i}$ during the registration phase.

\section{Performances evaluation}

In this section, we analyze the efficiency of the proposed approach. In particular, we have performed intensive simulations to evaluate the performances of the proposed solution, which we have compared with some reviewed approaches in Section 2, namely, $\mathrm{P}^{2} \mathrm{R}^{2} \mathrm{TM}$ [21], LPS for LBS [19], CMIX [10] and CARAVAN [15]. In what follows, we present the simulation environment, the performance metrics, and finally, we discuss the obtained results.

\subsection{Simulation environment and parameters}

The set of simulations are developed using the programming language Java, which we have provided with realistic vehicular mobility traces generated by SUMO (Simulation of Urban Mobility) [23. We consider a simulation scenario, which consists in generating a traffic in an urban area of $1500 \mathrm{~m} \times 1500 \mathrm{~m}$ derived from OpenStreetMap [24. The output traces of the mobility generator SUMO detail the location of every vehicle in the network at every time step along with their mobility profiles (i.e., speed, current lane, etc.). The generated traces are then used as inputs of the developed simulator. The simulation duration is of 1000s and the obtained results are averaged over 50 runs of simulation. Each vehicle is embedded with a pool of 1040 initial pseudonyms to use at each newly trip. When a vehicle passes by a RSU, it requests a new pseudonym to preserve its location privacy. These pseudonyms are generated by RSUs deployed at fixed positions through the simulation area. The main parameters considered in our simulations are summarized in Table 2 .

With respect to the reviewed approaches, the performance measurements are performed in the same simulation environment as for the proposed solution. Moreover, 


\begin{tabular}{|l|l|}
\hline Parameter & Value \\
\hline \hline Simulation area & $1500 \mathrm{~m} \times 1500 \mathrm{~m}$ \\
\hline Simulation duration & $1000 \mathrm{~s}$ \\
\hline Number of vehicles & $50,100,150,200$ and 250 \\
\hline Vehicle communication range & $250 \mathrm{~m}$ \\
\hline Number of RSUs $(n)$ & $7,11,15,19$ and 23 \\
\hline RSU communication range & $1000 \mathrm{~m}$ \\
\hline Pseudonym pool size $(N)$ & 1040 \\
\hline Initial pseudonym $p_{i k}^{(0)}$ size & 32 byte \\
\hline Individual pseudonym $p_{i}^{(j)}$ size & 56 byte \\
\hline ECC public and private keys & 28 byte \\
\hline Data transmission rate & 6 Mbit/s \\
\hline
\end{tabular}

Table 2: Simulation parameters

we set some parameter values according to the cryptographic techniques used and the operating of each protocol. For instance, in CMIX [10], we consider the creation of cryptographic mix zones at 4 road intersections defined by a transmission range $R_{C M I X}$ of $400 \mathrm{~m}$. All vehicles within a mix zone obtain a symmetric key of 32 byte to encrypt their messages while within the zone. In CARAVAN [15], each vehicle is preloaded with a set of 1040 pseudonyms which are used to send safety messages every 300ms. Therefore, the largest period of silence to update pseudonyms is fixed to the time interval between two safety message broadcasts. In LPS for LBS [19], each vehicle periodically (i.e., every $300 \mathrm{~ms}$ ) broadcasts a beacon of 100 byte with navigational data (e.g., its location, average speed, direction, etc.) needed for group maintenance. Note here that we consider, for all the protocols described earlier, RSA public and private keys of 2048 bits, which have the same security level as 224-bit ECC keys [25]. In $\mathrm{P}^{2} \mathrm{R}^{2} \mathrm{TM}$ [21], each RSU broadcasts its segment list, which holds the identifier of each segment it handles. According to the simulation area, we consider that each RSU has on the average 4 segments, where each segment has an identifier of 5 byte. Besides, each vehicle has a set of 1040 certified ECC public and private key pairs.

\subsection{Performance metrics}

To evaluate the efficiency of the proposed approach, we were interested on three important performance metrics, namely, the storage load, the communication load, and the response time. Through the storage load, we evaluate how well the proposed approach deals with the resources limitation issue in VNs. In fact, the usage of resourceconstrained devices managing cryptographic materials must be taken in charge when designing privacy-preserving solutions for VNs. The storage load is estimated by the total amount of bytes stored in both OBUs and RSUs. Through the communication load, we quantify how well the proposed approach optimizes vehicular communications. Reducing the number of exchanged messages allows to meet with the unique constraints of VNs in terms of high vehicles mobility and intermittent connectivity, as well as to prevent the performance degradation of ITS applications, which may result from bandwidth reduction due to frequent pseudonym changing requests. The communication load is 
computed from the total amount of bytes exchanged by OBUs and RSUs. Through the response time metric, we analyze how well the proposed approach satisfies the real-time requirement of ITS applications in terms of delays, which is of the utmost importance especially in safety-related applications. In fact, the sooner a vehicle receives information about traffic conditions, the more time it will have to react properly.

\subsection{Traffic density impact}

In this subsection, we discuss the road traffic density impact on the three performance metrics cited above.

Figure 4 illustrates the storage load in function of the vehicles number in the network. We note that the proposed solution achieves better results compared to the other protocols, in road traffic scenarios with more than 50 vehicles. Indeed, in the proposed solution, the OBU of each vehicle only needs to store a pool of 1040 initial pseudonyms, where each pseudonym is of 32 byte. This implies a slight increase of the storage load as the vehicles number rises. With regards to the other protocols, CARAVAN [15] presents a high increase of the storage load as each OBU is preloaded with a pseudonym pool, in addition to public and private key pairs, and public key certificates of 256 byte for each corresponding pseudonym. In LPS for LBS [19], we note that the storage load is increasingly high due to the different keys that need to be stored. In fact, each OBU obtains a public and private key pair during registration, in addition to all group members and group leader public keys of 256 byte, which are used for LBS requests encryption in the shuffling process.

Figure 5 illustrates the communication load in function of the vehicles number. Regarding the obtained results, the proposed solution shows better performances compared to the other protocols. In fact, in the proposed solution, each vehicle only sends a request of 64 byte when passing by a RSU within its communication range to ask for a new pseudonym. Each RSU generates then a pseudonym of 56 byte, which will be sent to the vehicle and the TA for conditional unlinkability and liability. Unlike the other protocols, this results in a slight increase of the communication load as the network becomes denser. In fact, in $\mathrm{P}^{2} \mathrm{R}^{2} \mathrm{TM}$ [21] for instance, each RSU present at road intersection broadcasts a message of 226 byte to disseminate its segment list to the passing vehicles. Upon reception, each vehicle needs to report its encrypted routes of 512 byte, along with a signature of 56 byte and a public key certificate of 60 byte. The RSUs issue then TMC traffic guidance information of 184 byte to the reporting vehicles in each segment.

Figure 6 illustrates the response time in function of the vehicles number. The obtained results show that the proposed solution offers better performances in comparison to the other protocols with a response time of 980 millisecond in high traffic density case. In CMIX [10, each vehicle within communication range of the RSU of a mix zone broadcasts a request to obtain the symmetric key that will be used to encrypt all safety messages until leaving the zone. Moreover, vehicles that are unable to obtain the symmetric key directly from the RSU can leverage on vehicles that are already in the mix zone to forward the key, which implies higher response time with vehicles number increase. In LPS for LBS [19], each vehicle that wants to access a location-based service doubly encrypts its request to prevent an adversary from relating the vehicle identity to the requests it has sent. In fact, each vehicle encrypts its request with the group 


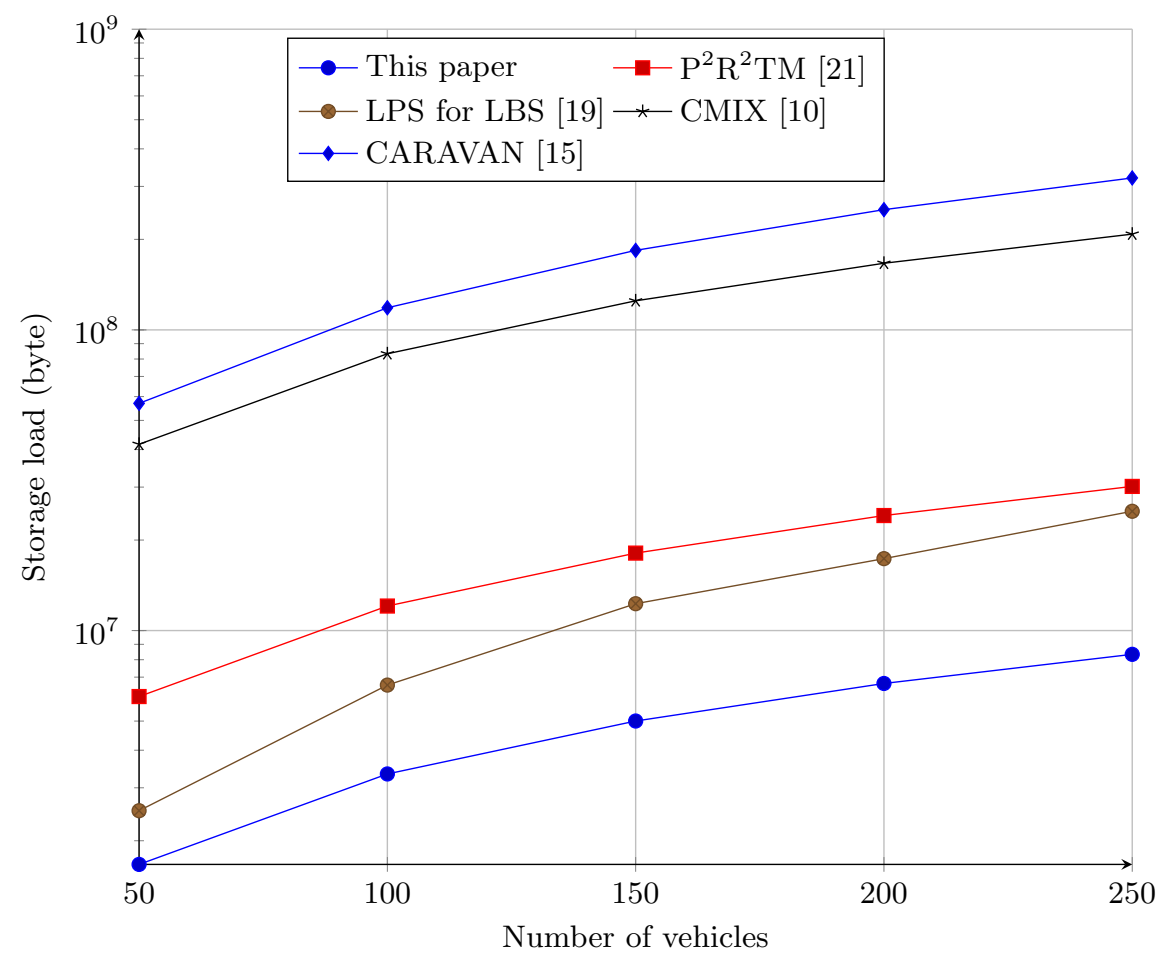

Figure 4: Storage load in function of the vehicles number

leader public key, then randomly chooses a group member to encrypt the latter message with the corresponding public key. The group leader waits then to receive all the members requests before aggregating and sending them to the LBS through the neighboring RSU. This implies a considerable response time that continues to increase with the network size. The obtained results show the effectiveness of the proposed solution for ITS applications even in high traffic density scenarios.

\subsection{Infrastructure deployment impact}

In this subsection, we discuss the impact of infrastructure deployment on previously cited performance metrics. The simulations were conducted in presence of 100 vehicles traveling through the considered area.

Figure 7 illustrates the storage load in function of the deployed RSUs number. We note an unnoticeable increase of the storage load as the RSUs number rises. However, the obtained results show that the proposed solution achieves better performances compared to the other protocols. In fact, each RSU deployed along a road section must store an ECC public and private key pair, and an encryption key share of 28 byte upon threshold group formation. Hence, the basic storage load for each RSU is of 84 byte, which results in a slight increase of the total storage load unlike the other protocols. For instance, in CMIX [10, each RSU must store a public and private key pair and its corresponding certificate, which raises their storage load to 768 byte. Moreover, as the number of deployed RSUs increases, their mix zones tend to overlap. Therefore, vehicles passing through must store the symmetric keys of 32 byte, which correspond to co-located zones and enable all safety messages decryption. 


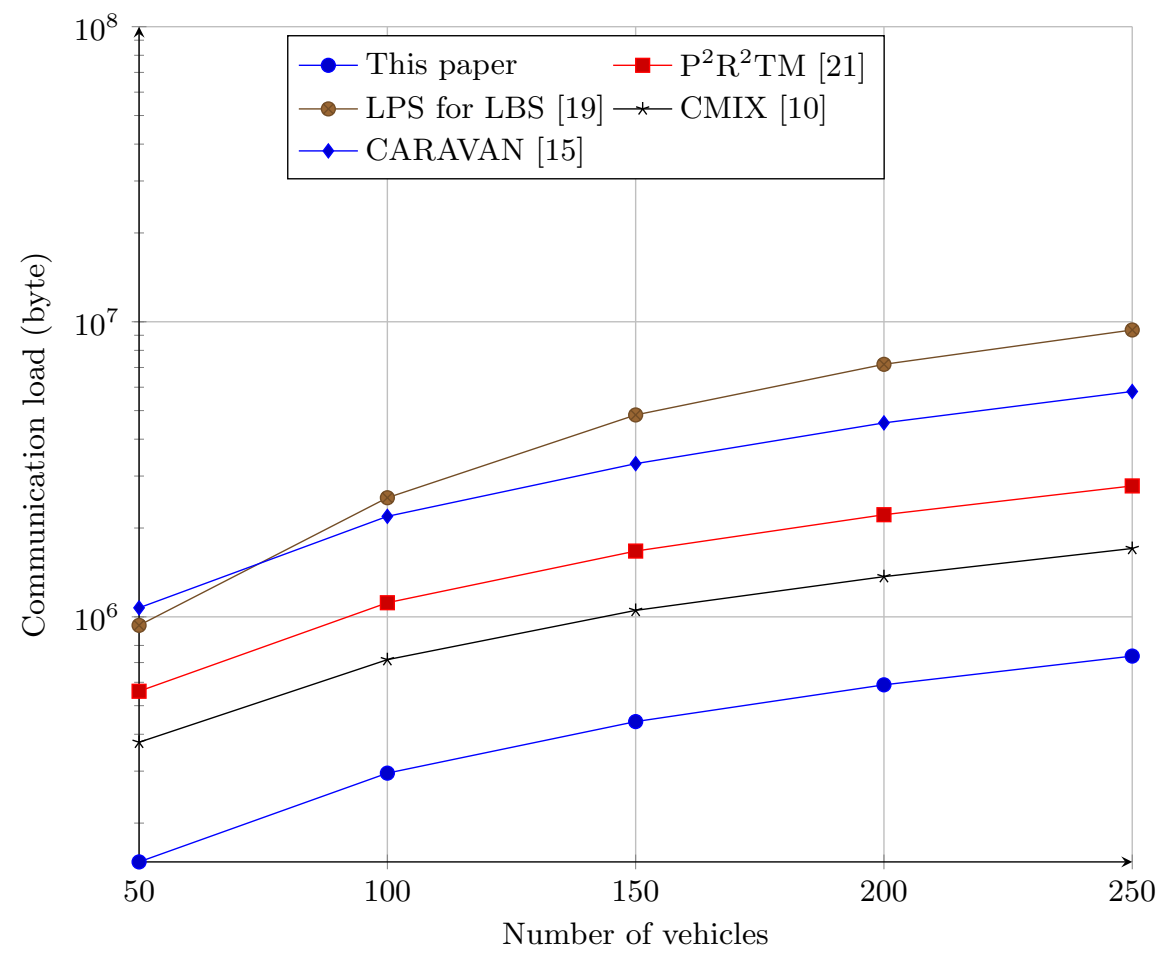

Figure 5: Communication load in function of the vehicles number

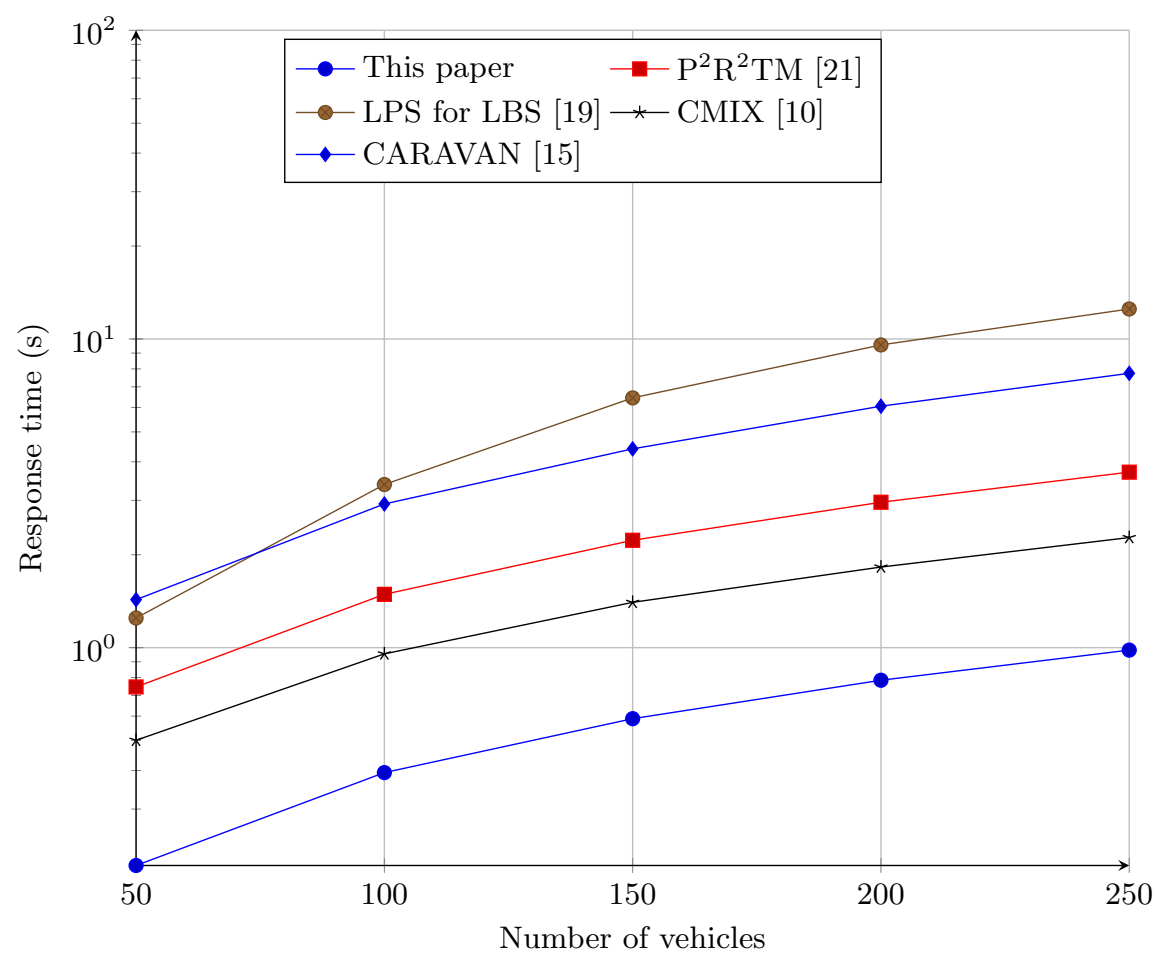

Figure 6: Response time in function of the vehicles number 
Figure 8 illustrates the communication load in function of the RSUs number. Regarding the obtained results, the proposed solution offers better performances compared to the other protocols. Indeed, in the proposed solution, each time a vehicle passes by a RSU during its trip, it requests a pseudonym to preserve its location privacy. Each RSU generates then the new pseudonym and sends it to the concerned vehicle and to the TA for prospective dispute resolution. This results in a slight increase of the total communication load as the number of deployed RSUs rises. Likewise, in $\mathrm{P}^{2} \mathrm{R}^{2} \mathrm{TM}$ [21], each RSU queries all the passing vehicles about whether they will take the segments it is responsible for, and sends their aggregated routes to the TMC. Each RSU broadcasts then traffic guidance information to the reporting vehicles, which decide whether to stay in the same route or take an alternative one. This implies a considerable increase of the communication load in crowded RSUs deployment scenarios.

Figure 9 illustrates the response time in function of the deployed RSUs number. The proposed solution shows better results in comparison to the other protocols with a response time of 625 millisecond when deploying 23 RSUs in the simulation area. In $\mathrm{P}^{2} \mathrm{R}^{2} \mathrm{TM}[21$, each RSU must wait to receive a certain number of encrypted routes to verify their authenticity and integrity. Each RSU aggregates then the encrypted routes and sends them to the TMC for vehicles guidance, hence, implying increasing response time. In LPS for LBS [19] and CARAVAN [15, even if the deployed RSUs number has no impact on the response time, the obtained results remain considerably high compared to the proposed solution, with a response time going up to $3.41 \mathrm{~s}$ and $3.73 \mathrm{~s}$, respectively, in dense RSUs deployment case. Regarding the obtained results, the proposed solution shows suitability for ITS applications, especially in some urban environments where a dense RSUs deployment may be needed to ensure vehicular communications, overcome signal propagation issues due to road obstacles (e.g., buildings, trees, etc.), and enhance collision reporting delays to the emergency services in low traffic density scenarios.

\subsection{Vehicles speed and mobility impact}

VNs undergo high dynamic topology and short connection duration due to vehicles quasi-permanent mobility. Hence, this inherent characteristic should be considered when dealing with VNs as it might have an impact on designed protocols performances. However, in the proposed approach, we do not consider vehicles speed as it has not an impact on the smooth pseudonym changing strategy operation. In fact, a vehicle cannot leave the coverage zone of an RSU without entering in it. Hence, the proposed pseudonym changing process is performed each time a vehicle passes by an RSU, and varying vehicles speed does not affect the amount of exchanged messages nor stored cryptographic parameters required by the protocol. Indeed, as regards to the storage load, each vehicle only stores its current used pseudonym and a pool of $N$ initial pseudonyms embedded in its OBU during the registration phase, whereas each RSU has a pre-stored public and private key pair, and an encryption key share received during the threshold-based key distribution phase performed when any vehicle requests a new pseudonym. On the other hand, a vehicle requests a new pseudonym whenever it belongs to the communication range of an RSU, which does not depend on the vehicle speed. Moreover, the proposed approach relies on ECC, which uses smaller key sizes than other public key cryptosystems thereby reducing processing overhead. Hence, the execution time of the pseudonym generation process can be neglected compared to a 


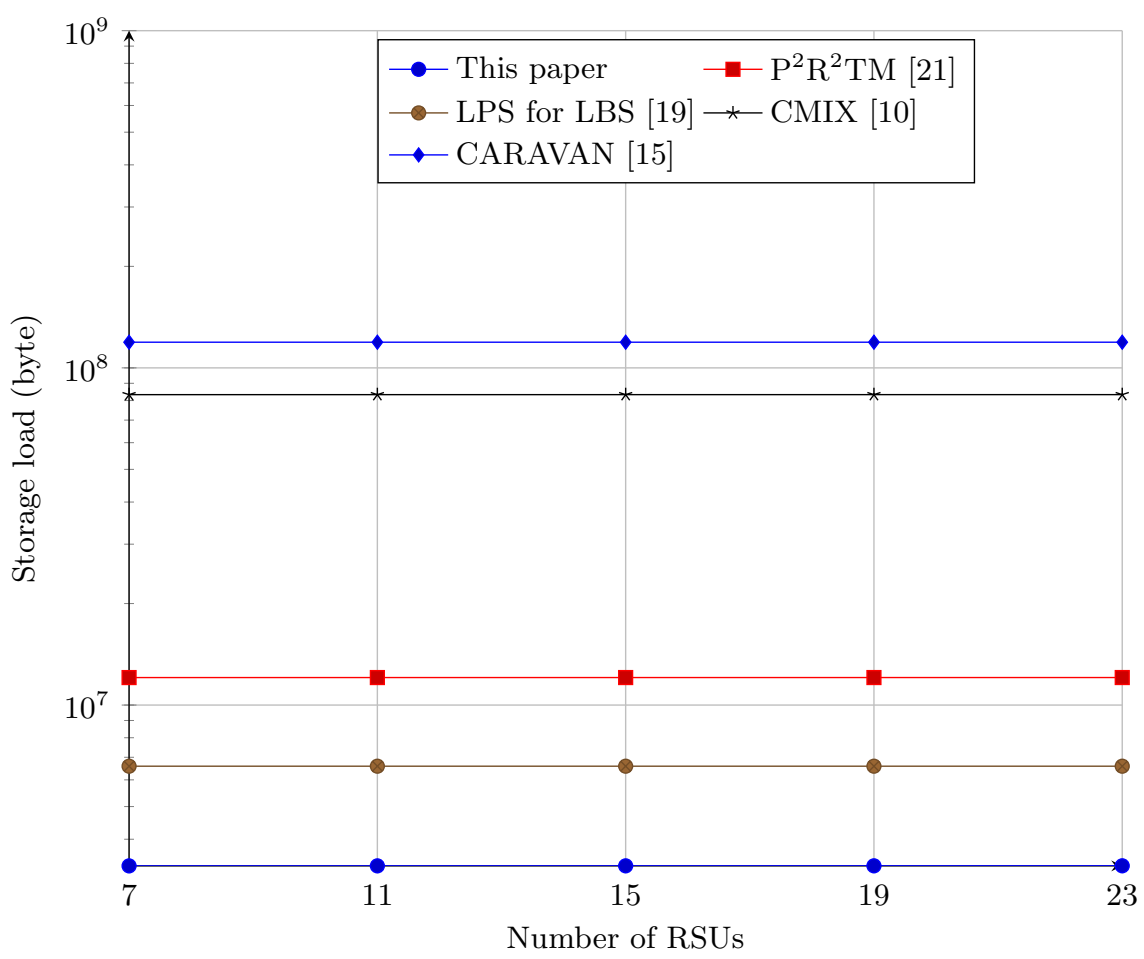

Figure 7: Storage load in function of the RSUs number

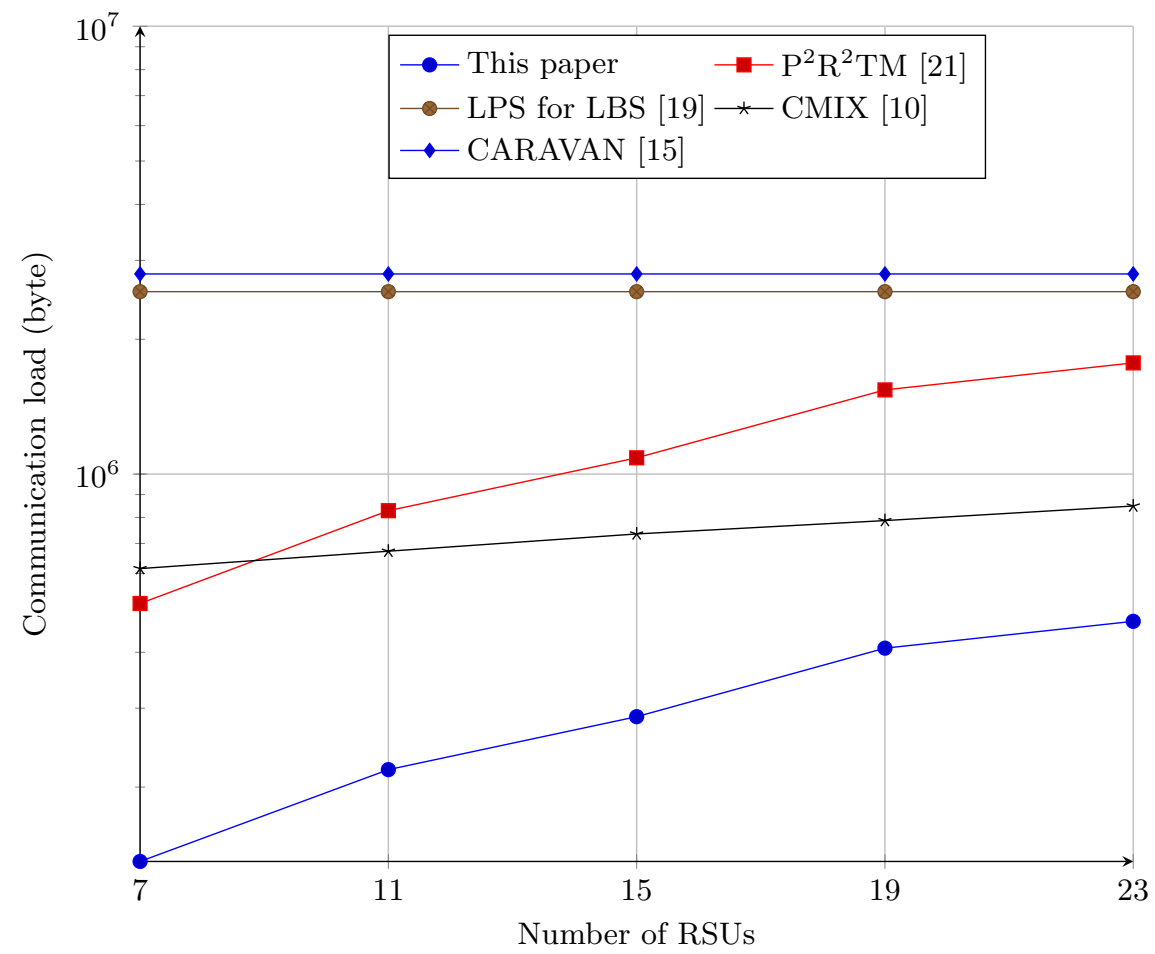

Figure 8: Communication load in function of the RSUs number 


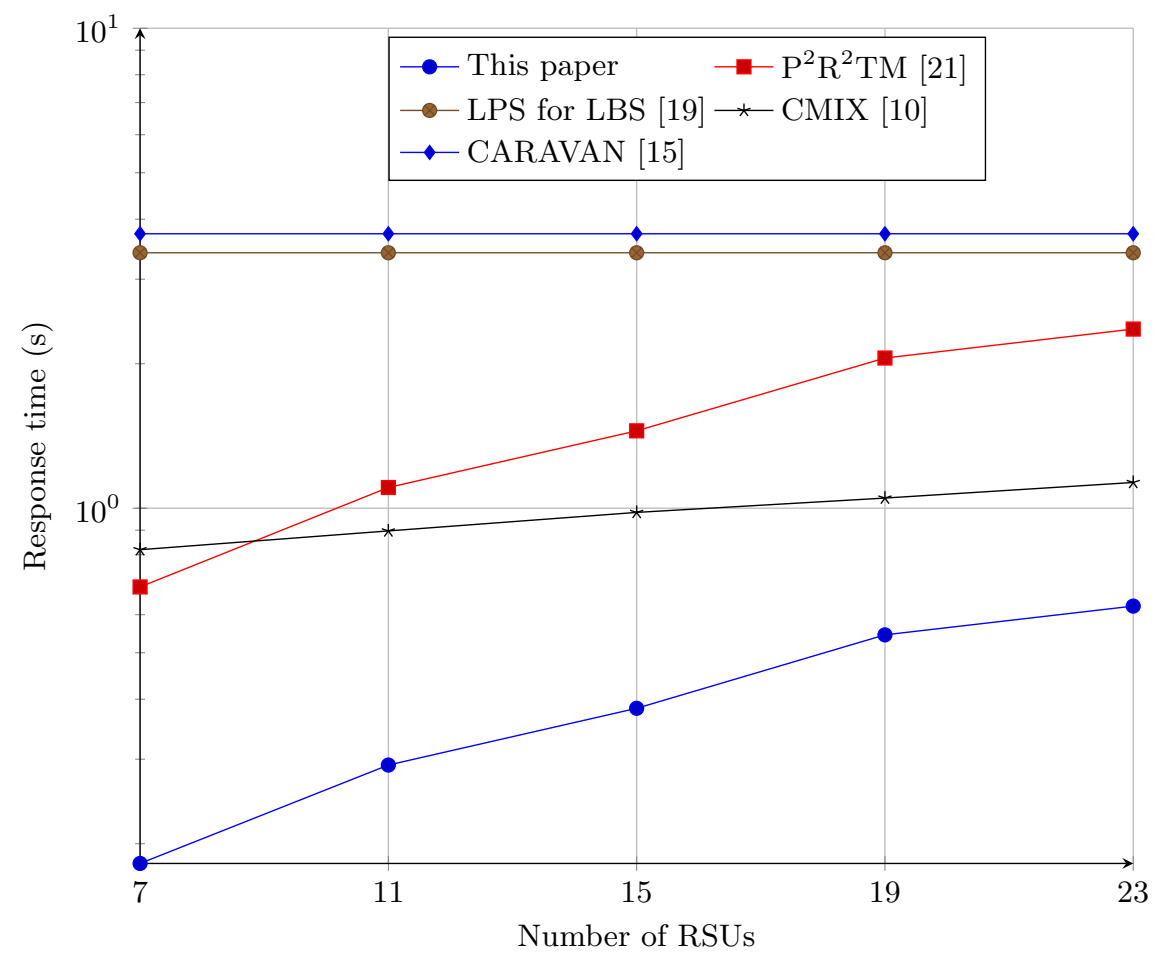

Figure 9: Response time in function of the RSUs number

vehicle traveling time inside an RSU coverage zone of $1000 \mathrm{~m}$, as vehicles speed goes from $30 \mathrm{~km} / \mathrm{h}$ to $50 \mathrm{~km} / \mathrm{h}$ in urban roads and agglomerations, while limited to $120 \mathrm{~km} / \mathrm{h}$ in highways.

\section{Conclusion}

Over the last decade, vehicular networks have known emerging interest from the research community and the automotive industry due to their key role in the design of promising applications for Intelligent Transportation Systems. Location privacy is a challenging issue in vehicular networks as many of these applications require vehicles to periodically send geolocation data including their position, velocity and direction. In this paper, we have proposed an effective pseudonymization approach to preserve location privacy in vehicular networks. The proposed approach relies on Vehicle-toInfrastructure communications and combines threshold cryptography properties to provide moving vehicles with new pseudonyms. The proposed approach provides a trade-off between efficiency and privacy protection. We have shown its resilience against some privacy threats and well-known security attack scenarios. We have also evaluated its efficiency through intensive simulations and compared it to the existing approaches. The obtained results indicate better performances of the proposed approach in terms of storage load, communication overhead and response time, which is suitable for Intelligent Transportation Systems applications even in congested road traffic scenarios and dense infrastructure deployment environments. 


\section{Acknowledgment}

This work was carried out in the framework of the research activities in the Computer Science Department, which is affiliated to the Faculty of Exact Sciences of the University of Bejaia.

\section{References}

[1] J. Wang, C. Jiang, K. Zhang, T. Q. Quek, Y. Ren and L. Hanzo, Vehicular sensing networks in a smart city: principles, technologies and applications, IEEE Wireless Communications 25(1), pp. 122-132, 2018.

[2] C. Bila, F. Sivrikaya, M-A. Khan and S. Albayrak, Vehicles of the Future: A survey of research on safety issues, IEEE Transactions on Intelligent Transportation Systems 18(5), pp. 1046-1065, 2017.

[3] F. Sakiz and S. Sen, A survey of attacks and detection mechanisms on intelligent transportation systems: VANETs and IoV, Ad Hoc Networks 61, pp. 33-50, 2017.

[4] H. Hasrouny, A. E. Samhat, C. Bassil and A. Laouiti, VANET security challenges and solutions: A survey, Vehicular Communications 7, pp. 7-20, 2017.

[5] F. Dressler, F. Kargl, J. Ott, O. K. Tonguz and L. Wischhof, Research challenges in inter-vehicular communication: lessons of the 2010 dagstuhl seminar, IEEE Communications Magazine 49(5), pp. 158-164, 2011.

[6] K. Ito, G. Hirakawa, Y. Arai and Y. Shibata, A road condition monitoring system using various sensor data in vehicle-to-vehicle communication environment, International Journal of Space-Based and Situated Computing 6(1), pp. 21-30, 2016.

[7] J. Chen, G. Mao, C. Li, W. Liang and D. G. Zhang, Capacity of cooperative vehicular networks with infrastructure support: multi-user case, IEEE Transactions on Vehicular Technology 67(2), pp. 1546-1560, 2018.

[8] D. Jia and D. Ngoduy, Enhanced cooperative car-following traffic model with the combination of V2V and V2I communication, Transportation Research Part B: Methodological 90, pp. 172-191, 2016.

[9] S. Djahel, N. Jabeur, R. Barrett and J. Murphy, Toward V2I communication technology-based solution for reducing road traffic congestion in smart cities, In IEEE International Symposium on Networks, Computers and Communications (ISNCC), Yasmine Hammamet, Tunisia, IEEE, pp. 1-6, 2015.

[10] J. Freudiger, M. Raya, M. Félegyházi, P. Papadimitratos and J-P. Hubaux, Mixzones for location privacy in vehicular networks, In Proceedings of the 1st ACM International Workshop on Wireless Networking For Intelligent Transportation Systems (WiN-ITS), Vancouver, Canada, ACM, 2007. 
[11] L. Buttyàn, T. Holczer and I. Vajda, On the effectiveness of changing pseudonyms to provide location privacy in VANETs, In Proceedings of the 4th European Conference on Security and Privacy in ad hoc and sensor networks, Cambridge, UK, Springer Berlin Heidelberg, pp. 129-141, 2007.

[12] A. Boualouache, S-M. Senouci and S. Moussaoui, VLPZ: The vehicular location privacy zone, Procedia Computer Science, Vol. 83, pp. 369-376, 2016.

[13] R. Lu, X. Lin, T. H. Luan, X. Liang and X. Shen, Pseudonym changing at social spots: An effective strategy for location privacy in VANETs, IEEE Transactions on Vehicular Technology 61(1), pp. 86-96, 2012.

[14] B. Ying, D. Makrakis and Z. Hou, Motivation for protecting selfish vehicles location privacy in vehicular networks, IEEE Transactions on Vehicular Technology 64(12), pp. 5631-5641, 2015.

[15] K. Sampigethaya, L. Huang, M. Li, R. Poovendran, K. Matsuura and K. Sezaki, CARAVAN: Providing location privacy for VANET, In Proceedings of the 3rd Workshop on Embedded Security in Cars (ESCAR), Cologne, Germany, 2005.

[16] W. Zhang, S. Jiang, X. Zhu, Y. Wang, Cooperative downloading with privacy preservation and access control for value-added services in VANETs, International Journal of Grid and Utility Computing 7(1), pp. 50-60, 2016.

[17] L. Buttyán, T. Holczer, A. Weimerskirch and W. Whyte, SLOW: A practical pseudonym changing scheme for location privacy in VANETs, In Proceedings of the 1st IEEE Vehicular Networking Conference (VNC), Tokyo, Japan, IEEE, pp. $1-8,2009$.

[18] M. Li, K. Sampigethaya, L. Huang and R. Poovendran, Swing \& swap: Usercentric approaches towards maximizing location privacy, In Proceedings of the 5th ACM Workshop on Privacy in Electronic Society, Alexandria, VA, ACM, pp. 19-28, 2006.

[19] S. Doumiati, T. Al Choikani and H. Artail, LPS for LBS: Location-privacy scheme for location-based services, In Proceedings of the 11th IEEE International Conference on Computer Systems and Applications, Doha, Qatar, IEEE, pp. 449-456, 2014.

[20] Y. Mei, G. Jiang, W. Zhang and Y. Cui, A collaboratively hidden location privacy scheme for VANETs, International Journal of Distributed Sensor Networks 10(3), 2014.

[21] K. Rabieh, M. M. Mahmoud and M. Younis, Privacy-preserving route reporting schemes for traffic management systems, IEEE Transactions on Vehicular Technology 66(3), pp. 2703-2713, 2017.

[22] W. Yun and L. Dianjun, An efficient threshold signature scheme based on the elliptic curve cryptosystem, In Proceedings of the IEEE International Conference on Computer Science and Electronics Engineering, Hangzhou, China, IEEE, pp. 349$352,2012$. 
[23] Simulation of Urban Mobility (SUMO), http://sumo.sourceforge.net, Accessed August 24, 2018.

[24] OpenStreetMap, http://www.openstreetmap.org, Accessed August 24, 2018.

[25] D. Mahto, D. A. Khan and D. K. Yadav, Security Analysis of Elliptic Curve Cryptography and $R S A$, In Proceedings of the World Congress on Engineering, London, UK, pp. 419-422, 2016. 
Siham Bouchelaghem received the Master Degree in 2016 in Computer Science from the University of Bejaia, (Algeria). She is currently a $\mathrm{PhD}$ student in the Computer Science Department in the University of Bejaia, after winning the first place in the $\mathrm{PhD}$ entrance examination. Her research interests include security and privacy in Smart Cities, vehicular networks, and autonomous vehicles.

Mawloud Omar is currently an Assistant Professor with the University of Bejaia, Algeria, where he is a member of the Research Unit LaMOS. He received the Engineering Diploma from the University of Chlef, (Algeria), in 2004, and the Magister and PhD Degrees in Computer Science from the University of Bejaia, (Algeria), respectively in 2007 and 2011. 